\title{
A study of nonlinear variable viscosity in finite-length tube with peristalsis
}

\author{
Y. Abd Elmaboud ${ }^{\mathrm{a}, \mathrm{b}, *}$, Kh.S. Mekheimer ${ }^{\mathrm{c}, \mathrm{d}}$ and Sara I. Abdelsalam ${ }^{\mathrm{e}}$ \\ ${ }^{a}$ Mathematics Department, Faculty of Science and Arts, Khulais, King Abdulaziz University (KAU), Jeddah, KSA \\ ${ }^{\mathrm{b}}$ Mathematics Department, Faculty of Science, Al-Azhar University (Assiut Branch), Assiut, Egypt \\ ${ }^{\mathrm{c}}$ Mathematics \& Statistic Department, Faculty of Science, Taif University, Taif, Saudi Arabia \\ ${ }^{\mathrm{d}}$ Mathematics Department, Faculty of Science, Al-Azhar University, Nasr City, Cairo, Egypt \\ ${ }^{\mathrm{e}}$ Basic Science Department, Faculty of Engineering, The British University in Egypt, Al-Shorouk City, \\ Cairo, Egypt
}

\begin{abstract}
Peristaltic motion of an incompressible Newtonian fluid with variable viscosity induced by periodic sinusoidal traveling wave propagating along the walls of a finite-length tube has been investigated. A perturbation method of solution is sought. The viscosity parameter $\alpha(\alpha<<1)$ is chosen as a perturbation parameter and the governing equations are developed up to the first-order in the viscosity parameter $(\alpha)$. The analytical solution has been derived for the radial velocity at the tube wall, the axial pressure gradient across the length of the tube, and the wall shear stress under the assumption of low Reynolds number and long wavelength approximation. The impacts of physical parameters such as the viscosity and the parameter determining the shape of the constriction on the pressure distribution and on the wall shear stress for integral and non-integral number of waves are illustrated. The main conclusion that can be drawn out of this study is that the peaks of pressure fluctuate with time and attain different values with non-integral numbers of peristaltic waves. The considered problem is very applicable in study of biological flow and industrial flow.
\end{abstract}

Keywords: Peristalsis, finite tube, variable viscosity, lubrication theory

\section{Introduction}

The peristaltic transport is traveling contraction wave along a tube-like structure, and it results physiologically from neuron-muscular properties of any tubular smooth muscle. Peristalsis is now well-known to the physiologists to be one of the major mechanisms for fluid transport in many biological systems. In particular, a peristaltic mechanism may be involved in swallowing food through the esophagus, urine transport from kidney to bladder through the ureter. The study of the mechanism of peristalsis of Newtonian and non-Newtonian fluids has recently become the object of scientific research. Since the first investigation of Latham [1], several theoretical and experimental

\footnotetext{
*Corresponding author: Y. Abd Elmaboud, Tel.: +9665382 39670; E-mail: yass_math@yahoo.com.
}

attempts have been made to understand peristaltic action in different situations. The earliest models of peristaltic pumping assumed trains of periodic sinusoidal waves in infinitely long two-dimensional channels or axisymmetric tubes. Such models fall into two classes: $(i)$ a model developed by Fung and Yih [2] which is restricted to small amplitudes but has no restrictions on Reynolds number; and (ii) the lubrication-theory model introduced by Shapiro et al. [3]. Shapiro et al. [3] theoretically examined the peristaltic flow of viscous fluid induced by sinusoidal wall propagation under the assumptions of long wavelength and low Reynolds number. Zein and Ostrach [4] studied the peristalsis of urine flow in human ureters with long wave approximation. Ramachandra and Usha [5] studied the peristalsis of the peripheral-layer immiscible fluids in a circular tube under long-wavelength and low-Reynolds number assumptions. Some authors 
(Abd El Naby and El Misiery [6]; Abd El Naby et al. [7]; Mekheimer [8]) studied peristaltic flow of Newtonian fluid with constant/variable viscosity under the effect of magnetic field or through a porous medium. Further, some progress have been made in studying peristalsis of non-Newtonian fluids in a channel or a tube, we refer to some interesting studies in the references (Siddiqui and Schwarz [9]; Tsiklauri and Beresnev [10]; Hayat et al. [11]; Hayat et al. [12]; Abd Elmaboud and Mekheimer [13]; Mekheimer and Abdel-Wahab [14]). Mekheimer et al. [15] investigated the peristaltic motion of incompressible Newtonian fluid in a planar channel. Previous studies of peristaltic transport have universally considered periodic peristaltic waves in infinite tubes, ignoring the inherently non-steady effects associated with the finite-length tubes encountered in real peristaltic pumps and in physiological peristaltic flows. Physiologically, transport by a single bolus moving along a tube of finite length is of particular interest as occurs in the esophagus, for instance. The effect of variable viscosity on the peristaltic flow of a Newtonian fluid in a uniform cylindrical tube has been discussed by Abd Elnaby and El Shamy [16]. They considered viscosity function as linear in half width of the channel as Srivastava et al. [17]. Li and Brasseur [18] presented a model for unsteady peristaltic transport of incompressible Newtonian fluid, but not with variable viscosity. Therefore, the aim of this article is to study peristalsis induced by periodic sinusoidal traveling wave propagating along the walls of a finite-length tube of a Newtonian incompressible fluid with variable viscosity. Keeping this in view, the mathematical model is constructed to study the influence of the viscosity and the parameter determining the shape of constriction in swallowing of food bolus (water) through the esophagus. The food bolus is supposed to be viscous fluid and the geometry of the wall surface of the esophagus is considered as peristaltic wave. It was noticed that the lubrication-theory approximations remain applicable in the present investigation, since the radius of the small esophagus is very small compared with the wavelength. The solutions of the momentum equations are given for small Reynolds number and long wavelength approximations. A comparison of our results with others shows that the results in the present work are more general than the results obtained by Li and Brasseur [18]. Moreover, the results obtained from Figs. 2 and 5 are similar to those given by Tripathi et al. [19] who found that for integral number of waves the ratio of the relaxation to the retardation time for a Jeffrey fluid decreases the magnitude of the pressure difference. We formulate the problem in Sect. 2. In Sect. 3, we solve the problem. The graphical results and discussion as well as the conclusions are presented in Sects. 4 and 5, respectively.

\section{Formulation of the problem and mathematical model}

In this paper, the flow of an incompressible Newtonian fluid with variable viscosity through a finite-length tube of length $L^{\prime}$ with peristalsis is considered. The wall motion is supposed to be created by waves propagating along the tube wall (Fig. 1) which are mathematically modeled as

$$
h^{\prime}\left(Z^{\prime}, t^{\prime}\right)=a+b \cos ^{2}\left(\frac{\pi}{\lambda}\left(Z^{\prime}-c t^{\prime}\right)\right),
$$

where $h^{\prime}$ represents the transverse vibration of the wall, $a$ is the average radius of the bolus, $b$ is the amplitude of the wave, $\lambda$ is the wavelength and $c$ is wave velocity.

The problem has been studied in cylindrical coordinate system $\left(R^{\prime}, Z^{\prime}\right)$, where the $Z^{\prime}$-axis is taken along the axis of the tube while $R^{\prime}$ is taken along the radial.

The equations governing the motion of an incompressible Newtonian fluid with variable viscosity in the laboratory frame, are given by

$$
\begin{gathered}
\frac{\partial V^{\prime}}{\partial R^{\prime}}+\frac{\partial U^{\prime}}{\partial Z^{\prime}}+\frac{V^{\prime}}{R^{\prime}}=0, \\
\rho\left[\frac{\partial V^{\prime}}{\partial t^{\prime}}+V^{\prime} \frac{\partial V^{\prime}}{\partial R^{\prime}}+U^{\prime} \frac{\partial V^{\prime}}{\partial Z^{\prime}}\right] \\
=-\frac{\partial P^{\prime}}{\partial R^{\prime}}+\frac{\partial}{\partial R^{\prime}}\left[2 \mu^{\prime}\left(R^{\prime}\right)\left(\frac{\partial V^{\prime}}{\partial R^{\prime}}\right)\right] \\
+\frac{\partial}{\partial Z^{\prime}}\left[\mu^{\prime}\left(R^{\prime}\right)\left(\frac{\partial V^{\prime}}{\partial Z^{\prime}}+\frac{\partial U^{\prime}}{\partial R^{\prime}}\right)\right] \\
+\frac{2 \mu^{\prime}\left(R^{\prime}\right)}{R^{\prime}}\left(\frac{\partial V^{\prime}}{\partial R^{\prime}}-\frac{V^{\prime}}{R^{\prime}}\right), \\
\rho\left[\frac{\partial U^{\prime}}{\partial t^{\prime}}+V^{\prime} \frac{\partial U^{\prime}}{\partial R^{\prime}}+U^{\prime} \frac{\partial U^{\prime}}{\partial Z^{\prime}}\right] \\
=-\frac{\partial P^{\prime}}{\partial Z^{\prime}}+\frac{1}{R^{\prime}} \frac{\partial}{\partial R^{\prime}}\left[\mu ^ { \prime } ( R ^ { \prime } ) R ^ { \prime } \left(\frac{\partial V^{\prime}}{\partial Z^{\prime}}\right.\right. \\
\left.\left.+\frac{\partial U^{\prime}}{\partial R^{\prime}}\right)\right]+\frac{\partial}{\partial Z^{\prime}}\left[2 \mu^{\prime}\left(R^{\prime}\right)\left(\frac{\partial V^{\prime}}{\partial Z^{\prime}}+\frac{\partial U^{\prime}}{\partial R^{\prime}}\right)\right],
\end{gathered}
$$


with the boundary conditions

$$
\begin{aligned}
& \left.U^{\prime}\right|_{R^{\prime}=h^{\prime}}=0,\left.\quad V^{\prime}\right|_{R^{\prime}=h^{\prime}}=\frac{\partial h^{\prime}}{\partial t^{\prime}}, \\
& \left.\frac{\partial U^{\prime}}{\partial R^{\prime}}\right|_{R^{\prime}=0}=0,\left.\quad V^{\prime}\right|_{R^{\prime}=0}=0, \\
& \left.P^{\prime}\right|_{Z^{\prime}=0}=P_{0}^{\prime},\left.\quad P^{\prime}\right|_{Z^{\prime}=L^{\prime}}=P_{L^{\prime}}^{\prime},
\end{aligned}
$$

where $\rho$ is the density, $P^{\prime}$ is the pressure, $\mu^{\prime}\left(R^{\prime}\right)$ is the viscosity function, $U^{\prime}$ and $V^{\prime}$ are the axial and the radial velocities and $P_{i}^{\prime}, P_{L^{\prime}}^{\prime}$ are the uniform pressures applied to the inlet and the outlet of the tube $\left(Z=0, L^{\prime}\right)$, respectively. Consider the following non-dimensional variables and parameters:

$$
\begin{aligned}
& R=\frac{R^{\prime}}{a}, Z=\frac{Z^{\prime}}{\lambda}, U=\frac{U^{\prime}}{c}, V=\frac{\lambda V^{\prime}}{a c}, \\
& P=\frac{a^{2}}{\lambda \mu_{0} c} P^{\prime}, \mu(R)=\frac{\mu^{\prime}\left(R^{\prime}\right)}{\mu_{0}}, \\
& L=\frac{L^{\prime}}{\lambda}, \phi=\frac{b}{a}, R e=\frac{\rho c a}{\mu}, \\
& \delta=\frac{a}{\lambda}, \quad h=\frac{h^{\prime}}{a}=1+\phi \cos ^{2} \pi(Z-t),
\end{aligned}
$$

where $\phi$ is the amplitude ratio, $R e$ is the Reynolds number, $\delta$ is the dimensionless wave number. To proceed, we non-dimensionalize Equations (2.2-2.5), this yields

$$
\frac{1}{R} \frac{\partial}{\partial R}(R V)+\frac{\partial U}{\partial Z}=0,
$$

$$
\begin{aligned}
\operatorname{Re} \delta^{3} & {\left[\frac{\partial V}{\partial t}+V \frac{\partial V}{\partial R}+U \frac{\partial V}{\partial Z}\right] } \\
= & -\frac{\partial P}{\partial R}+\delta^{2} \frac{\partial}{\partial R}\left[2 \mu(R)\left(\frac{\partial V}{\partial R}\right)\right] \\
& +\delta^{2} \frac{\partial}{\partial Z}\left[\mu(R)\left(\delta^{2} \frac{\partial V}{\partial Z}+\frac{\partial U}{\partial R}\right)\right] \\
& +\delta^{2} \frac{2 \mu(R)}{R}\left(\frac{\partial V}{\partial R}-\frac{V}{R}\right), \\
\operatorname{Re} \delta & {\left[\frac{\partial U}{\partial t}+V \frac{\partial U}{\partial R}+U \frac{\partial U}{\partial Z}\right] } \\
= & -\frac{\partial P}{\partial Z}+\frac{1}{R} \frac{\partial}{\partial R}\left[\mu(R) R\left(\delta^{2} \frac{\partial V}{\partial Z}+\frac{\partial U}{\partial R}\right)\right] \\
& +\delta \frac{\partial}{\partial Z}\left[2 \mu(R)\left(\delta^{2} \frac{\partial V}{\partial Z}+\frac{\partial U}{\partial R}\right)\right] .
\end{aligned}
$$

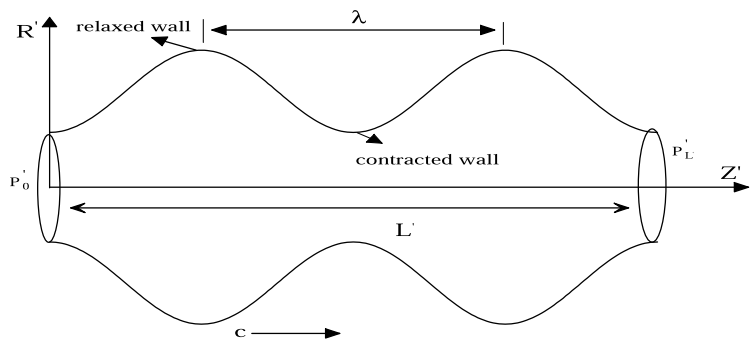

Fig. 1. Schematic diagram of the problem.

The dimensionless boundary conditions will be in the form

$$
\begin{aligned}
& \left.U\right|_{R=h}=0,\left.\quad V\right|_{R=h}=\frac{\partial h}{\partial t}, \\
& \left.\frac{\partial U}{\partial R}\right|_{R=0}=0,\left.\quad V\right|_{R=0}=0, \\
& \left.P\right|_{Z=0}=P_{i},\left.\quad P\right|_{Z=L}=P_{L} .
\end{aligned}
$$

To reduce the problem, we will use the lubrication theory approximations of infinitesimally small wall curvature $(\delta \rightarrow 0)$ and Reynolds number $(\operatorname{Re} \rightarrow 0)$. The approximations assume that inertial effects are negligible and that the dominant axial scale is much larger than the dominant radial scale. In this limit, pressure is uniform on each cross-section and the governing equations and boundary conditions become

$$
\begin{gathered}
\frac{1}{R} \frac{\partial}{\partial R}(R V)+\frac{\partial U}{\partial Z}=0, \\
0=-\frac{\partial P}{\partial R} \\
0=-\frac{\partial P}{\partial Z}+\frac{1}{R} \frac{\partial}{\partial R}\left[\mu(R) R\left(\frac{\partial U}{\partial R}\right)\right], \\
\left.U\right|_{R=h}=0,\left.\quad V\right|_{R=h}=\frac{\partial h}{\partial t}, \\
\left.\frac{\partial U}{\partial R}\right|_{R=0}=0,\left.\quad V\right|_{R=0}=0, \\
\left.P\right|_{Z=0}=P_{i},\left.\quad P\right|_{Z=L}=P_{L} .
\end{gathered}
$$

For the present problem, we choose the variation of the viscosity model in the dimensionless form as: $\mu(R)=$ $1-\alpha R^{n}$, where $\alpha(\alpha<1)$ is viscosity parameter and $n(\geq 2)$ is the parameter determining the shape of the constriction [16]. Substituting the value of $\mu(R)$ in the Equation (2.13) we get 


$$
0=-\frac{\partial P}{\partial Z}+\frac{1}{R} \frac{\partial}{\partial R}\left[\left(1-\alpha R^{n}\right) R\left(\frac{\partial U}{\partial R}\right)\right]
$$

\section{Method of solution}

It is evident that Equation (2.15) can be solved directly to get the solutions, but it is convenient for the present problem to expand the flow quantities in a power series of the small parameter $\alpha$ as follows:

$$
\begin{aligned}
& U=U_{0}+\alpha U_{1}+\alpha^{2} U_{2}+\ldots . \\
& V=V_{0}+\alpha V_{1}+\alpha^{2} V_{2}+\ldots . \\
& P=P_{0}+\alpha P_{1}+\alpha^{2} P_{2}+\ldots .
\end{aligned}
$$

If we substitute (3.1) into (2.11, 2.12, 2.14 and 2.15) and separate the terms of different order in $\alpha$, we obtain the following systems of partial differential equations together with boundary conditions as follows:

\section{System of order zero}

$$
\begin{gathered}
\frac{1}{R} \frac{\partial}{\partial R}\left(R V_{0}\right)+\frac{\partial U_{0}}{\partial Z}=0, \\
0=-\frac{\partial P_{0}}{\partial R} \\
0=-\frac{\partial P_{0}}{\partial Z}+\frac{1}{R} \frac{\partial}{\partial R}\left[R\left(\frac{\partial U_{0}}{\partial R}\right)\right],
\end{gathered}
$$

with the boundary conditions:

$$
\begin{aligned}
& \left.U_{0}\right|_{R=h}=0,\left.\quad V_{0}\right|_{R=h}=\frac{\partial h}{\partial t}, \\
& \left.\frac{\partial U_{0}}{\partial R}\right|_{R=0}=0,\left.\quad V_{0}\right|_{R=0}=0, \\
& \left.P_{0}\right|_{Z=0}=P_{i},\left.\quad P_{0}\right|_{Z=L}=P_{L} .
\end{aligned}
$$

\section{System of order one}

$$
\begin{gathered}
\frac{1}{R} \frac{\partial}{\partial R}\left(R V_{1}\right)+\frac{\partial U_{1}}{\partial Z}=0, \\
0=-\frac{\partial P_{1}}{\partial R}
\end{gathered}
$$

$$
0=-\frac{\partial P_{1}}{\partial Z}+\frac{1}{R} \frac{\partial}{\partial R}\left[R \frac{\partial U_{1}}{\partial R}-R^{n+1} \frac{\partial U_{0}}{\partial R}\right]
$$

with the boundary conditions:

$$
\begin{aligned}
& \left.U_{1}\right|_{R=h}=0,\left.\quad V_{1}\right|_{R=h}=0, \\
& \left.\frac{\partial U_{1}}{\partial R}\right|_{R=0}=0,\left.\quad V_{1}\right|_{R=0}=0, \\
& \left.P_{1}\right|_{Z=0}=0,\left.\quad P_{1}\right|_{Z=L}=0 .
\end{aligned}
$$

Solving the above sets of equations with the corresponding boundary conditions, we get

\section{Zero order solution}

$$
\begin{gathered}
U_{0}=\frac{1}{4} \frac{\partial P_{0}}{\partial Z}\left(R^{2}-h^{2}\right), \\
V_{0}=\frac{R}{4}\left[h \frac{\partial h}{\partial z} \frac{\partial P_{0}}{\partial Z}-\frac{\partial^{2} P_{0}}{\partial Z^{2}}\left(\frac{1}{4} R^{2}-\frac{1}{2} h^{2}\right)\right],
\end{gathered}
$$

Evaluating the radial velocity at the tube wall and using the condition $\left.V_{0}\right|_{R=h}=\frac{\partial h}{\partial t}$, we can get a relationship between the motion of the tube wall and the axial pressure gradient $\frac{\partial P_{0}}{\partial Z}$ as:

$$
\frac{\partial h}{\partial t}=\frac{h^{3}}{16} \frac{\partial^{2} P_{0}}{\partial Z^{2}}+\frac{h^{2}}{4} \frac{\partial h}{\partial Z} \frac{\partial P_{0}}{\partial Z} .
$$

Then, integrating Equation (3.12) from 0 to $Z$, the pressure gradient across the length of the tube is given by

$$
\begin{aligned}
\frac{\partial P_{0}}{\partial Z}= & \frac{1}{h^{4}(Z, t)}\left(G_{0}(t)\right. \\
& \left.+16 \int_{0}^{Z} h(S, t) \frac{\partial h(S, t)}{\partial t} d S\right),
\end{aligned}
$$

where $G_{0}(t)$ is a constant. Integrating Equation (3.13) from 0 to $Z$, the pressure difference is obtained as

$$
\begin{gathered}
P_{0}(Z, t)-P_{0}(0, t) \\
=G_{0}(t) \int_{0}^{Z} h^{-4}\left(S_{1}, t\right) d S_{1}+16 \int_{0}^{Z}\left(h^{-4}\left(S_{1}, t\right)\right. \\
\left.\quad \int_{0}^{S_{1}} h\left(S_{2}, t\right) \frac{\partial h\left(S_{2}, t\right)}{\partial t} d S_{2}\right) d S_{1} .
\end{gathered}
$$

By using the condition $\left.P_{0}\right|_{Z=L}=P_{L}$, with Equation (3.14) we can determine $G_{o}(t)$ as follows:

$$
G_{0}(t)=\frac{\left(P_{L}-P_{i}\right)-16 \int_{0}^{L}\left(h^{-4}\left(S_{1}, t\right) \int_{0}^{S_{1}} h\left(S_{2}, t\right) \frac{\partial h\left(S_{2}, t\right)}{\partial t} d S_{2}\right) d S_{1}}{\int_{0}^{L} h^{-4}\left(S_{1}, t\right) d S_{1}} .
$$



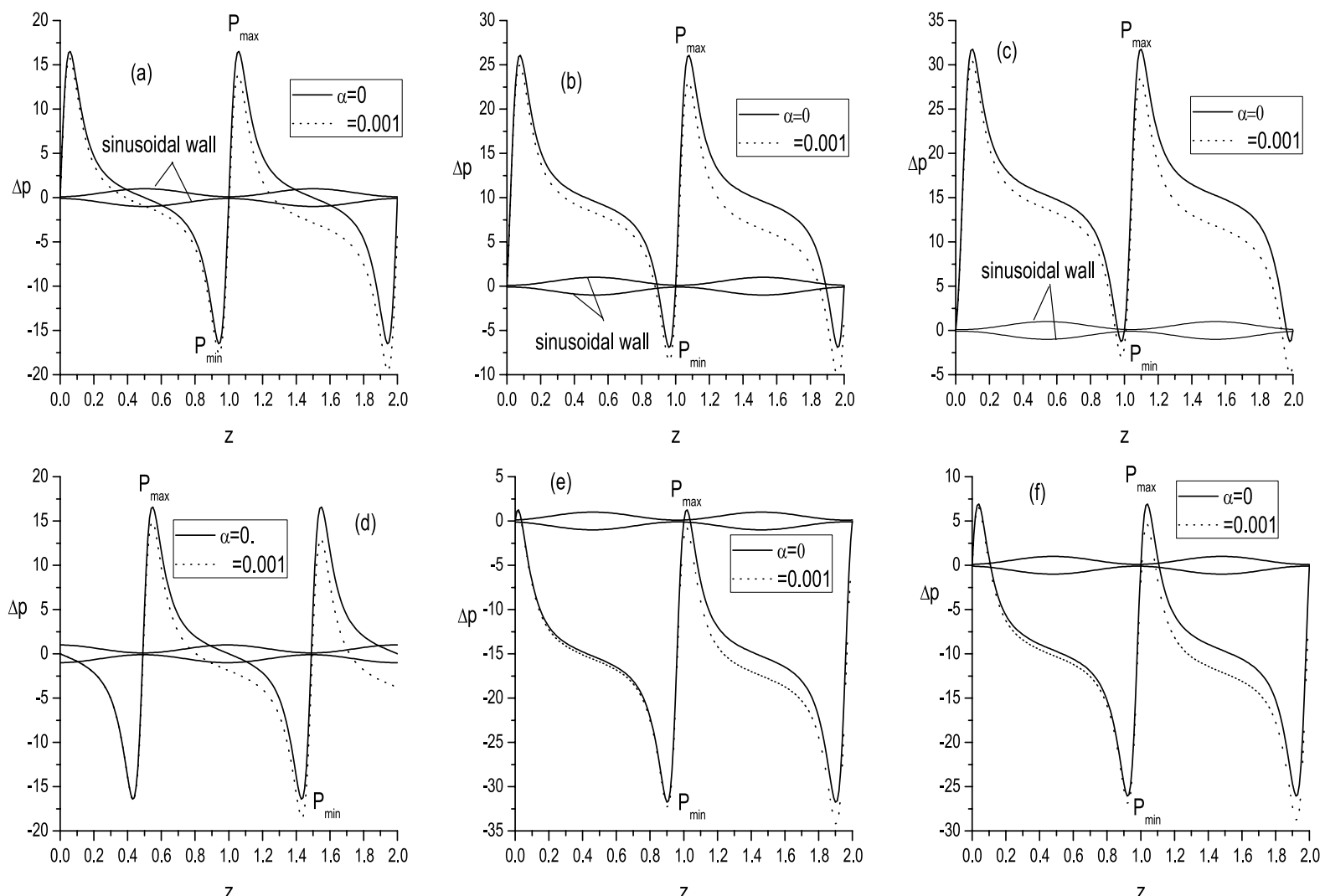

Fig. 2. Pressure distributions along the tube at six time instants in one wave period with two waves in the tube for: $(a) t=0,1$, $(b) t=$ $0.02 ;(c) t=0.04 ;(d) t=0.49 ;(e) t=0.96 ;(f) t=0.98$, with different values of $\alpha$ at $n=2, \phi=0.9$ and $L=2$.

The wall shear stress in this order given by

$$
\begin{aligned}
\tau_{0}= & \left.\frac{\partial U_{0}}{\partial R}\right|_{R=h}=\frac{1}{2 h^{3}(Z, t)}\left(G_{0}(t)\right. \\
& \left.+16 \int_{0}^{Z} h(S, t) \frac{\partial h(S, t)}{\partial t} d S\right) .
\end{aligned}
$$

\section{First order solution}

$$
\begin{aligned}
U_{1}= & \frac{1}{4} \frac{\partial P_{1}}{\partial Z}\left(R^{2}-h^{2}\right)+\frac{1}{2(n+2)} \frac{\partial P_{0}}{\partial Z}\left(R^{n+2}-h^{n+2}\right), \\
V_{1}= & \frac{1}{4} h R \frac{\partial h}{\partial Z} \frac{\partial P_{1}}{\partial Z}+\frac{R}{8} \frac{\partial^{2} P_{1}}{\partial Z^{2}}\left(h^{2}-\frac{r^{2}}{2}\right)+\frac{r}{4(n+2)} \\
& \frac{\partial^{2} P_{0}}{\partial Z^{2}}\left[h^{n+2}-\frac{2 r^{n+2}}{n+4}\right]+\frac{R h^{n+1}}{4} \frac{\partial h}{\partial Z} \frac{\partial P_{0}}{\partial Z} \cdot \text { (3.18) }
\end{aligned}
$$

To get the axial pressure gradient $\frac{\partial P_{1}}{\partial Z}$ in this order, we will evaluate the radial velocity $\left(V_{1}\right)$ at the tube wall and using the condition $\left.V_{1}\right|_{R=h}=0$, we get:

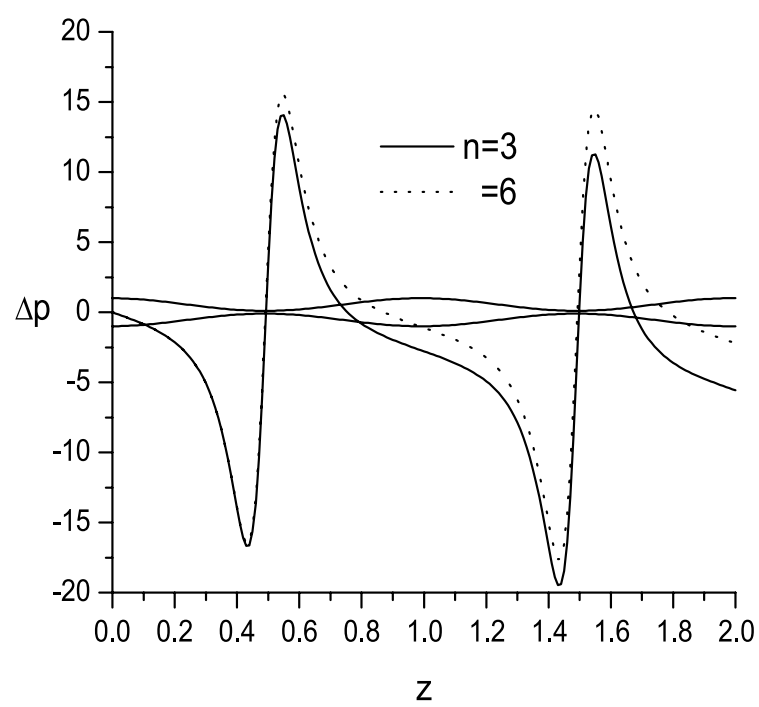

Fig. 3. Pressure distributions along the tube with different values of $n$ at $\alpha=0.2, t=0.49, \phi=0.9$ and $L=2$. 

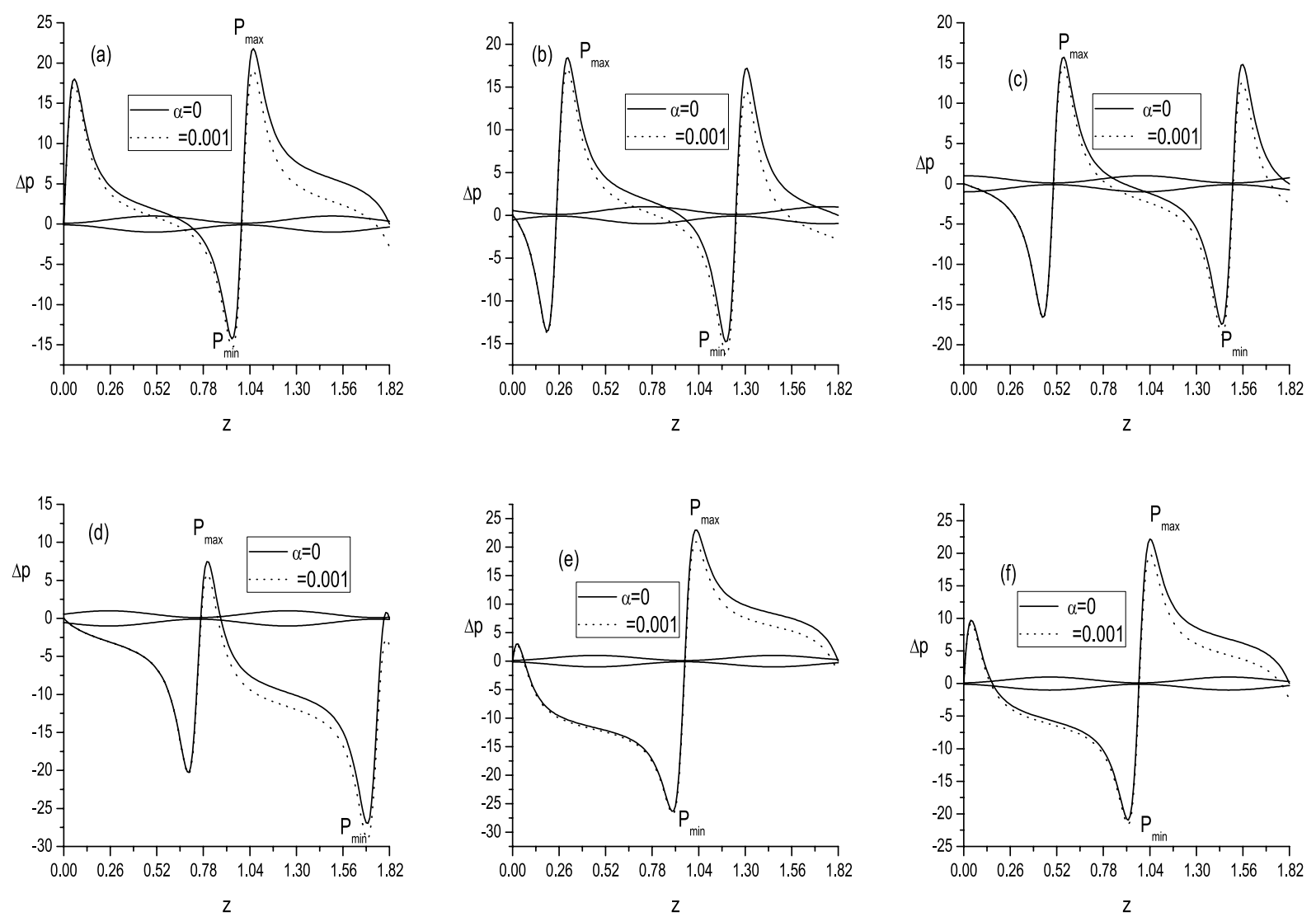

Fig. 4. Pressure distributions along the tube at six time instants with non-integral sinusoidal waves $L=1.82:(a) t=0$, (b) $t=0.02 ;(c) t=$ 0.04 ; (d) $t=0.49$; (e) $t=0.96$; (f) $t=0.98$, with different values of $\alpha$ at $n=2, \phi=0.9$.

$$
\begin{aligned}
& \frac{h^{2}}{4} \frac{\partial h}{\partial Z} \frac{\partial P_{1}}{\partial Z}+\frac{h^{3}}{16} \frac{\partial^{2} P_{1}}{\partial Z^{2}} \\
& +\frac{h^{n+3}}{4(n+4)} \frac{\partial^{2} P_{0}}{\partial Z^{2}}+\frac{h^{n+2}}{4} \frac{\partial h}{\partial Z} \frac{\partial P_{0}}{\partial Z}=0 .
\end{aligned}
$$

To proceed, we integrate Equation (3.19) from 0 to $Z$, the pressure gradient in this order across the length of the tube is given by

$$
\frac{\partial P_{1}}{\partial Z}=-\frac{16}{h^{4}}\left[\frac{h^{n+4}}{4(n+4)} \frac{\partial P_{0}}{\partial Z}+G_{1}(t)\right],
$$

where $G_{1}(t)$ is an integrating constant. Integrating Equation (3.20) along the tube from 0 to $Z$, the pressure difference is obtained as

$$
\begin{aligned}
P_{1}(Z, t)-P_{1}(0, t)= & -16\left[\frac{1}{4(n+4)} \int_{0}^{Z} h^{n}(S, t) \frac{\partial P_{0}}{\partial Z} d S\right. \\
& \left.+\frac{G_{1}(t)}{\int_{0}^{Z} h^{4}(S, t) d S}\right] .
\end{aligned}
$$

By using the conditions $\left.P_{1}\right|_{Z=L}=0$ and $\left.P_{1}\right|_{Z=0}=$ 0 , with Equation (3.21), we can determine $G_{1}(t)$ as follows:

$$
G_{1}(t)=-\frac{\int_{0}^{Z} h^{4}(S, t) d S}{4(n+4)} \int_{0}^{Z} h^{n}(S, t) \frac{\partial P_{0}}{\partial Z} d S .
$$

The wall shear stress in this order given by

$$
\tau_{1}=\left.\frac{\partial U_{1}}{\partial R}\right|_{R=h}=\frac{h}{2} \frac{\partial P_{1}}{\partial Z}+\frac{h^{n+1}}{2} \frac{\partial P_{0}}{\partial Z} .
$$

Analytical expressions given by Equations (3.14 and 3.21 ) represent the pressure difference $(\Delta p)$ between the inlet and any axial station $\mathrm{z}$.

\section{Numerical results and discussion}

In this section, we consider the finite tube length on transport characteristics. We have presented the numerical and computational results for analyzing the impact 

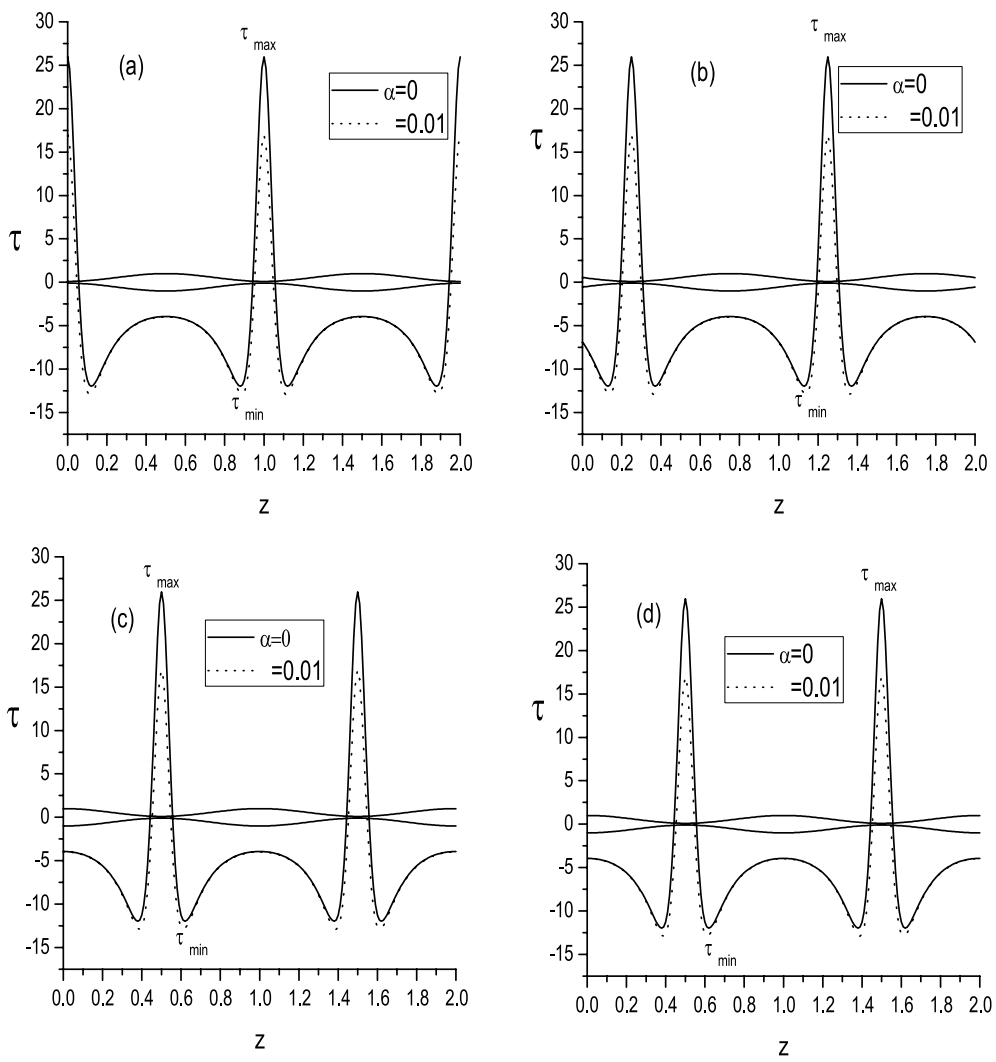

Fig. 5. Wall shear stress distribution at four time instants (a) $t=0,(b) t=0.25 ;(c) t=0.5 ;(d) t=0.75$, with different values of $\alpha$ at $n=4$, $\phi=0.9$ and $L=2$.

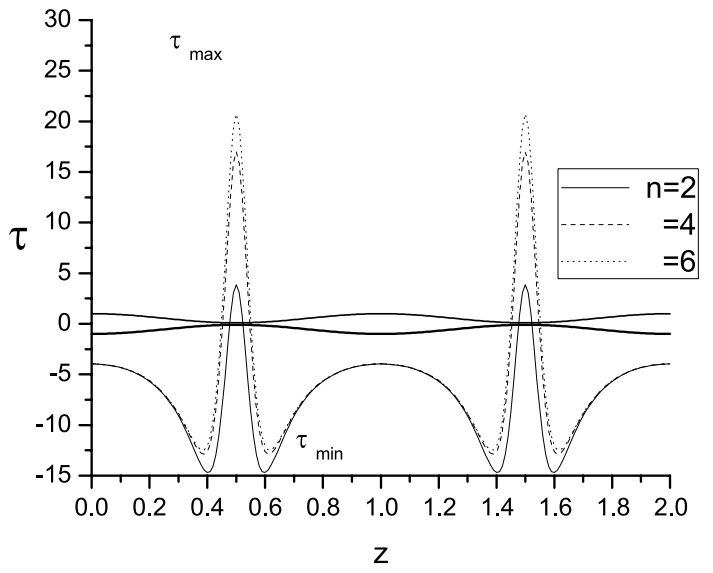

Fig. 6. Wall shear stress distribution with different values of $n$ at $\alpha=0.01, t=0.5, \phi=0.9$ and $L=2$.

of the parameters determining the viscosity parameter $\alpha$ and the shape of constriction $n$, on the local variables such as pressure distribution along the tube, $\Delta p$ and the shear stress distribution at the wall, $\tau$. For this objective, Figs. (2-7) have been plotted using computational programmes of Mathematica 7.0 software. One of the cases known as free pumping, i.e. pressures at both ends of the tube are zero $\left(P_{i}=P_{L}=0\right)$, is taken to discuss the characteristic of peristaltic flow pattern through the finite length of tube. This condition is rather important in the study that distinguishes the cases of finite length flow and infinite length tube flow. Notice that when the amplitude ratio $\phi$ is varied, the wave amplitude is adjusted to keep the volume of fluid within one wave fixed. In the figures, the solid and dotted lines are the pressure distributions along the tube, and the crossing middle solid lines are the corresponding wave shape. Figure $2 \mathrm{a}-\mathrm{f}$ depict the temporal examinations of the pressure distribution along the length of the tube at various instants, i.e. for discrete values of $t$ in the range $0.0-1.0$. In Fig. $2 \mathrm{a}(t=0)$, it is observed that the pressure tends to rise sharply at the inlet, reaches some peak, then falls at a lower rate at the middle of the bolus, comes down further to a lower trough and finally rises sharply to meet 

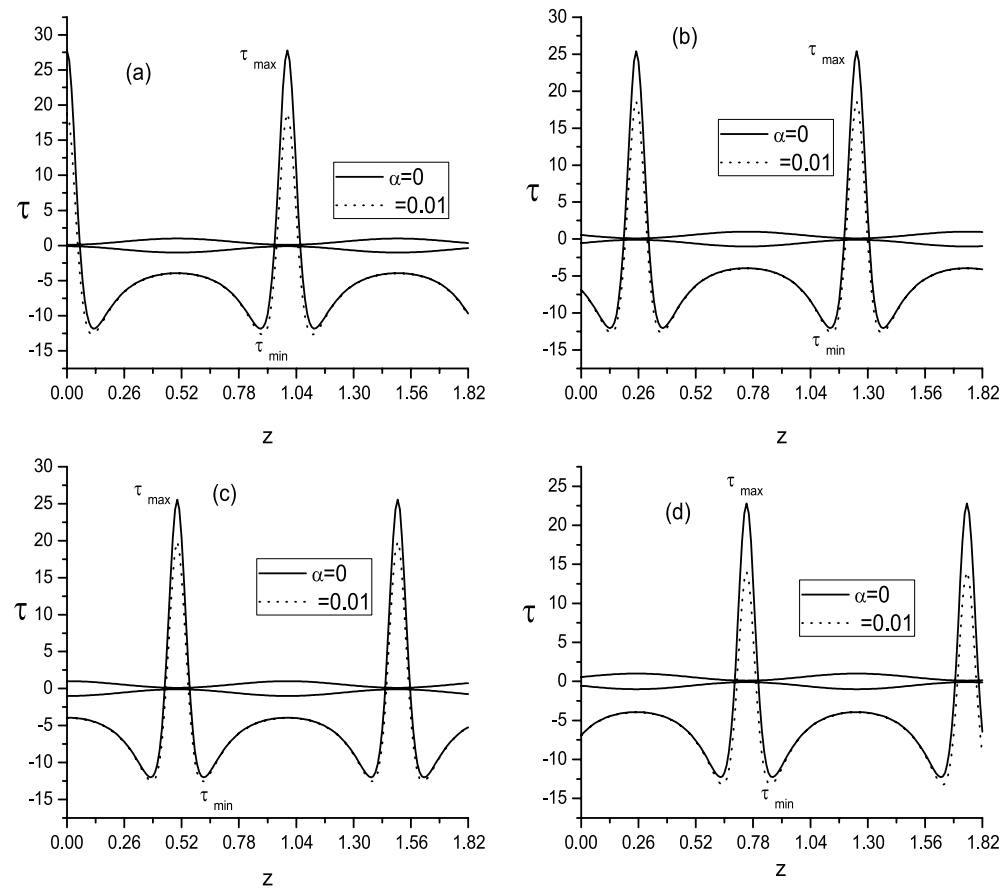

Fig. 7. Wall shear stress distribution at four time instants with non-integral sinusoidal waves $L=1.82$ : (a) $t=0$, (b) $t=0.25$; (c) $t=$ $0.5 ;(d) t=0.75$, with different values of $\alpha$ at $n=4$ and $\phi=0.9$.

the leading end of the bolus. The same distribution is repeated for the next bolus. After one fourth of the periodic cycle (Fig. 2d), the bolus moves ahead and a trailing bolus is on the way to entry. The graphs for higher values of $t$ represent a systematic progress of the boluses in the tube. Eventually, at the time $t=1$, which represents the completion of one period, the pressure distributions resembles that at $t=0$; this indicates that a new cycle is ready to set out (Fig. 2a). Further, we notice that within each peristaltic wave there exist two peaks in the pressure distribution with a gradual pressure ramp in between. The transition from a large negative peak to a large positive peak takes place at the point of minimum radius (the point of maximum occlusion) within the contraction zones. To the right of this point, the tube wall is moving radially inward $\left(\frac{\partial h}{\partial t}<0\right)$, creating a large gradient in pressure there. In the oesophagus, for instance, this inward motion of the tube wall is produced by the contraction of circular muscles within the oesophageal wall. To the left of this point of maximal occlusion, the tube wall is moving radially outward leading to a corresponding drop in pressure there. In Fig. 3, the same pressure distribution in one-wave period with two waves in the tube was studied for various values of the shape of con- striction $n$, i.e. throughout one time period. Temporal effects were found to be similar to those observed for different values of $\alpha$. Thus, they were excluded to avoid repetition. In Figs. 2 and 3, the effects of the parameters determining the viscosity parameter $\alpha$ and the shape of constriction $n$, on the pressure distribution along the length of the tube are presented. It is observed that the magnitude of the pressure along the length of the tube reduces with increasing the magnitude of $\alpha$ whereas it increases with increasing $n$. A similar observation is made for all values of $t$ ranging from $0-1$, i.e., throughout one-time period. Figures 4a-f elucidate the time-varying pressure distribution for a non-integral number of peristaltic waves in the tube where all the variables are the same as in Fig. 2 except for $L=1.82$. We define $\Phi$ as the relative difference in amplitude between the maximum pressure and minimum pressure, i.e. $\Phi=P_{\max }-P_{\min }$, which is invariant with time. When an integral number of waves occupies the tube, the peristaltic waves enter and leave the tube ends simultaneously, so the pressure waveform oscillates as a whole with a period equal to the period of a single wave. With a non-integral number of waves, the wave entering the tube is out of phase with the wave exiting the tube, leading to a shift in the 
waveforms between the two waves. In other words, it is observed that the peaks of pressure for the two different types of boluses are identical in the integral case while the peaks are different in the non-integral case. So that fluctuates with time only in the non-integral case. The effects of the physical parameters, $\alpha$ and $n$, do not reveal any difference in both cases. A relation between the local wall shear stress along the length of the tube at various instants and the axial distance is presented in Figs. 5-7. In Figs. 5 and 6, it is observed that the range between the peak and trough of local wall shear distribution decreases with an increase in the magnitude of $\alpha$ while enhances with an increase in the magnitude of $n$ for integral number of peristaltic waves. The graphs for higher values of $t$ represent a systematic progress for the stress distribution in the tube. It is further concluded that the temporal effects of the shear stress for different values of $\alpha$ are the same as those observed for different values of $n$. The figures of the latter case were excluded so as to avoid repetition. Figures 7a-7f depict the influence of viscosity parameter $\alpha$ on local wall shear stress along the length of the tube for non-integral number of peristaltic waves. It is revealed that the range between the peak and trough of local wall shear stress reduces with increasing the magnitude of $\alpha$.

\section{Conclusions}

In this study, we observe the inclusion of the parameters determining the viscosity $(\alpha)$ and the shape of constriction $(n)$, into the peristaltic flow of incompressible Newtonian fluid with variable viscosity through a finite-length tube. The problem is solved by means of a perturbation analysis with implementing the lubrication theory approximation of infinitesimally small wall curvature and Reynolds number. The considered problem is very applicable in study of biological flow and industrial flow. The main findings can be summarized as follows:

- The peaks of the pressure are identical in the integral number of waves propagating along the length of the tube, while the peaks are different in the non-integral number of waves propagating along the length of the tube.

- The physical parameters $\alpha$ and $n$ have the same effect on both the integral and non-integral number of waves.
- The range between the peak and trough of local wall shear stress reduces with increasing the magnitude of $\alpha$.

- The pressure distribution is the same at both times; $t=0$ and $t=1$.

- Unlike the infinite-tube model, fixed pressure at the ends induces fluctuations in pressure as the peristaltic waves pass through the tube inlet or outlet. Such fluctuations in pressure arise from the existence of non-integral number of peristaltic waves within the tube.

- A comparison of our results with others shows that the results in the present work are more general than the results obtained by $\mathrm{Li}$ and Brasseur [18].

\section{References}

[1] T.W. Latham, Fluid motion in a peristaltic pump, M.Sc. Thesis, MIT, Cambridge, MA, 1966.

[2] Y.C. Fung, C.S. Yih, Peristaltic transport, Trans ASME E: J AppL Mech 35 (1968), 669-675.

[3] A.H. Shapiro, M.Y. Jaffrin and S.L. Weinberg, Peristaltic pumping with long wavelength at low Reynolds number, J Fluid Mech 37 (1969), 799.

[4] T.F. Zien and S.A. Ostrach, A long wave approximation to peristaltic motion, J Biomech 3 (1970), 63.

[5] R.A. Ramachandra and S. Usha, Peristaltic transport of two immiscible viscous fluids in a circular tube, J Fluid Mech 298 (1995), 271.

[6] Abd El Hakeem Abd El Naby and A.E.M. El Misiery, Effects of an endoscope and generalized Newtonianfluid on peristaltic motion, Applied Mathematics and Computation 128 (2002), 19.

[7] Abd El Hakeem Abd El Naby, A.E.M. El Misery and I.I. El Shamy, Effects of an endoscope and fluid with variable viscosity on peristaltic motion, Applied Mathematics and Computation 158 (2004), 497.

[8] Kh.S. Mekheimer, Peristaltic transport of a Newtonian fluid through a uniform and non-uniform annulus, The Arabian $J$ for Sc and Eng 30(1A) (2005), 69.

[9] A.M. Siddiqui, W.H. Schwarz, Peristaltic flow of a secondorder fluid in tubes, Journal of Non-Newtonian Fluid Mechanics, 53 (1994), 257.

[10] D. Tsiklauri and I. Beresnev, Non-Newtonian effects in the peristaltic flow of a Maxwell fluid, Physical Review E 64 (2001), 036303.

[11] T. Hayat, A. Afsar, M. Khan and S. Asghar, Peristaltic transport of a third order fluid under the effect of a magnetic field, Comp and Math App 53 (2007), 1074.

[12] T. Hayat, M. Khan, A.M. Siddiqui and S. Asghar, Non-linear peristaltic flow of a non-Newtonian fluid under effect of a magnetic field in a planar channel, Communications in Nonlinear Science and Numerical Simulation 12 (2007), 910.

[13] Y. Abd Elmaboud and Kh.S. Mekheimer, Non-linear peristaltic transport of a second-order fluid through a porous medium, Applied Mathematical Modelling 35 (2011), 2695. 
[14] Kh.S. Mekheimer, A.N. Abdel-Wahab, Compressibility effects on peristaltic flow of a non-Newtonian Maxwell fluid through an annulus, Fluid Transport: Theory, Dynamics and Transport, ISBN: 978-1-61122-676-8, (2011) Nova Science Publishers, Inc.

[15] Kh.S. Mekheimer, E.F. El Shehawey and A.M. Elaw, Peristaltic motion of a particle-fluid suspension in a planar channel, Int $J$ Theor Phys 37 (1998), 2895.

[16] A.H. Abd Elnaby and I.I. El Shamy, Effects of inclined magnetic field on a fluid with nonlinear variable viscosity in a uniform tube with peristalsis, Multidiscipline Modeling in Mat And Str 5 (2009), 395-409.

[17] L.M. Srivastava, V.P. Srivastava and S.N. Sinha, Peristaltic transport of a physiological fluid. Part-I. Flow in non-uniform geometry, Biorheol 20 (1983), 153.

[18] M. Li and J.G. Brasseur, Non-steady peristaltic transport in finite-length tubes, J Fluid Mech 248 (1993), 129-151.

[19] D. Tripathi, N. Ali, T. Hayat, M.K. Chaube and A.A. Hendi, Peristaltic flow of MHD Jeffrey fluid through finite length cylendrical tube, Appl Math Mech Engl Ed 32 (2011), 1231. 

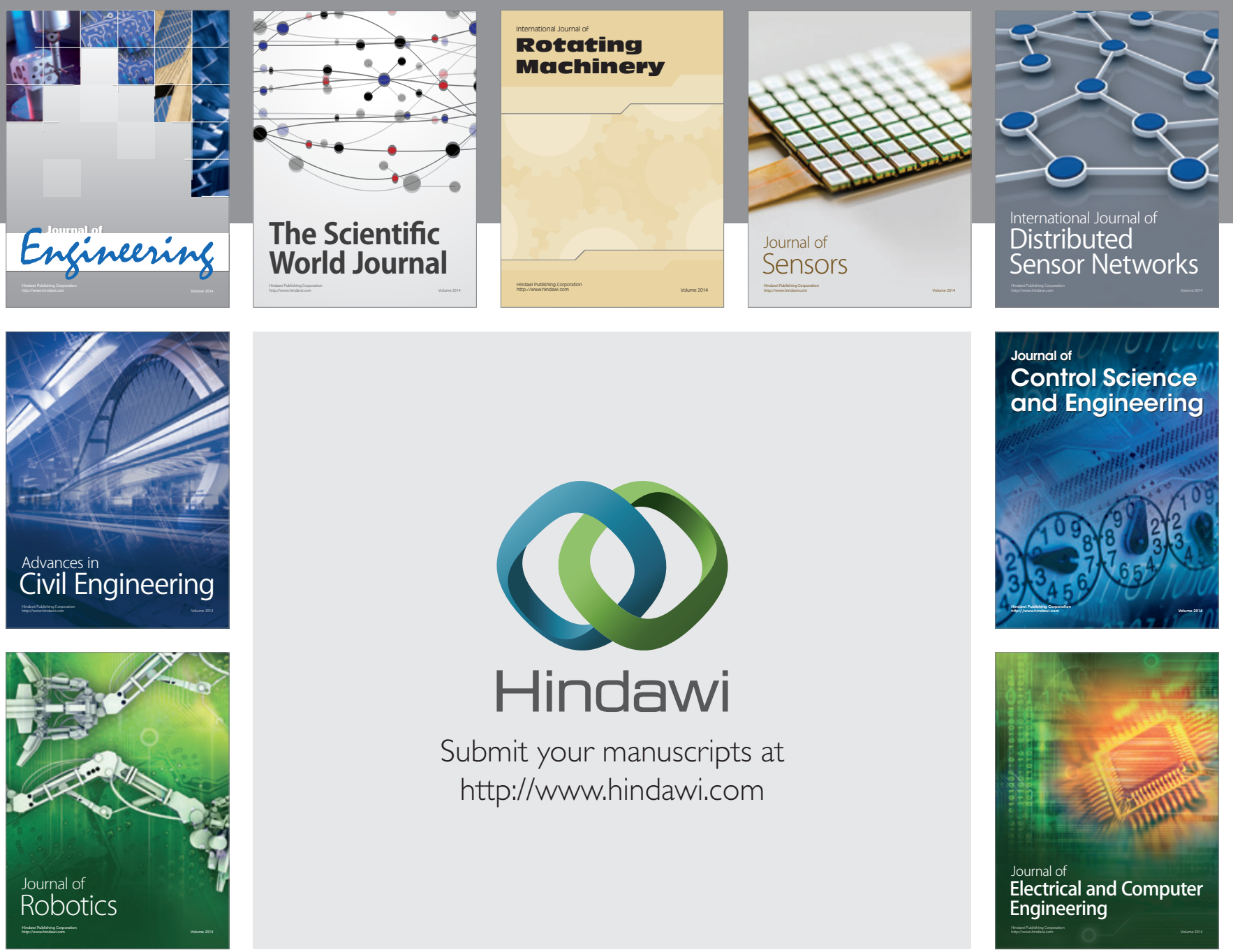

Submit your manuscripts at

http://www.hindawi.com
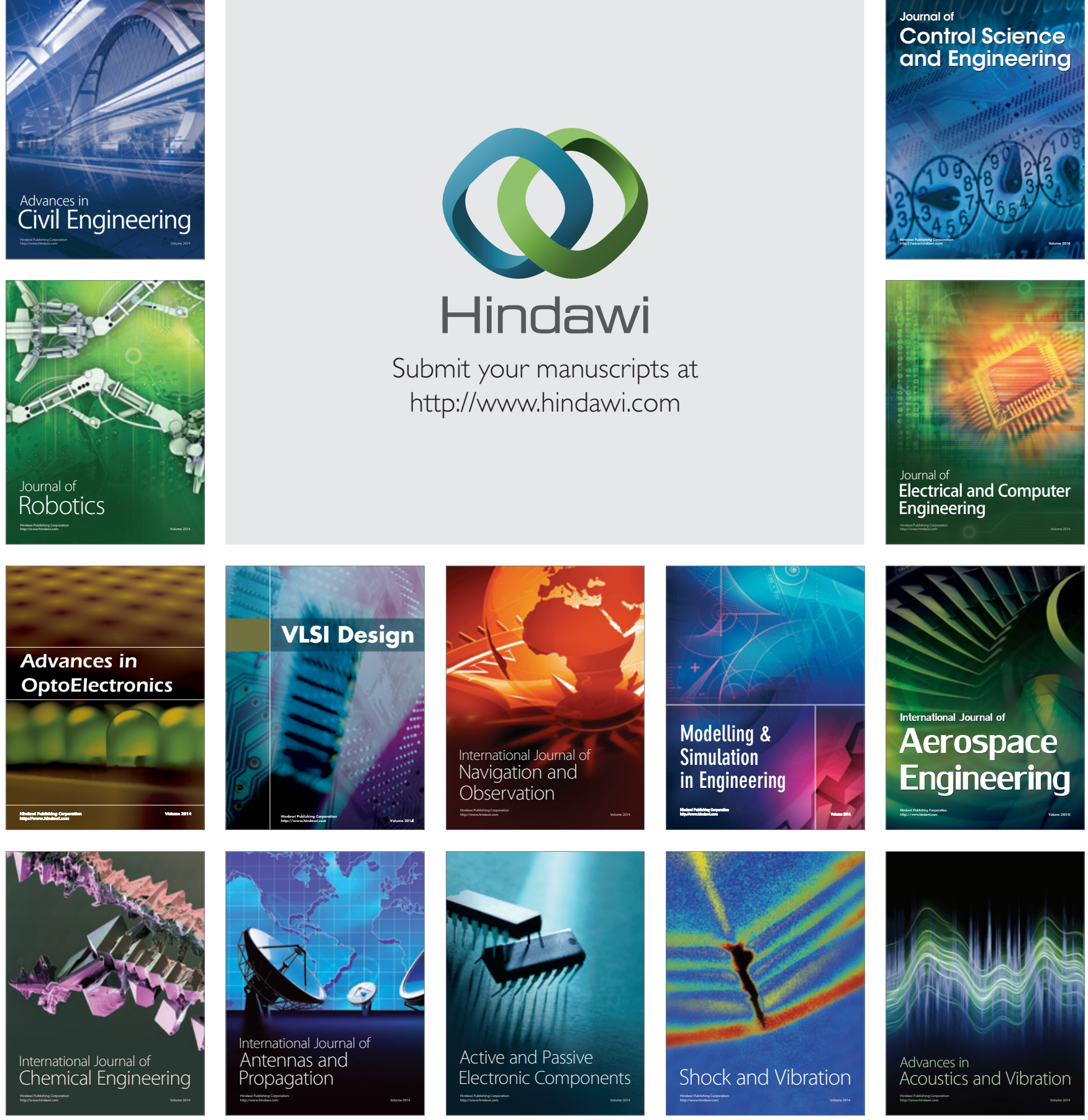